\title{
Management of cervical intraepithelial neoplasia in Japanese pregnant women
}

\author{
Yuko Matsubara, Toru Fujioka, Hisashi Hashimoto, Katsuyuki Hamada, Keiichi Matsubara, \\ Akihiro Nawa \\ Department of Obstetrics and Gynecology, School of Medicine, Ehime University, Toon, Japan \\ Email: takeyu@m.ehime-u.ac.jp \\ Received 7 February 2013; revised 10 March 2013; accepted 19 March 2013 \\ Copyright (C) 2013 Yuko Matsubara et al. This is an open access article distributed under the Creative Commons Attribution License, \\ which permits unrestricted use, distribution, and reproduction in any medium, provided the original work is properly cited.
}

\begin{abstract}
Objective: In this study, we evaluated the management of cervical intraepithelial neoplasia (CIN) in pregnant women and evaluated the postpartum prognosis. Methods: Twenty-four pregnant women, who were diagnosed with CIN at Ehime University Hospital between January 2008 and October 2012, were recruited. The mode of delivery and pathophysiological examination results in the postpartum period were evaluated. Results: Four patients were antenatally diagnosed with either CIN1 or CIN2. Of these patients, CIN regressed or remained stable during pregnancy, and there was no disease progression in the postpartum period. Nine patients were diagnosed with severe dysplasia (CIN3) and eleven patients had carcinoma in situ (CIS). Of these patients, 5/9 (55\%) and 4/11 (36\%), respectively, had disease regression postnatally. No CIN lesion progressed to invasive carcinoma after delivery. Conclusions: We determined that many cases diagnosed antenatally with CIN regressed during the postpartum period, regardless of the grade of CIN. We recommend a conservative management strategy with careful ante- and postpartum examinations for pregnant women with CIN.
\end{abstract}

Keywords: Cervical Intraepithelial Neoplasia; Prenancy

\section{INTRODUCTION}

Cervical carcinoma is the most common malignant tumor to be diagnosed during pregnancy, with a maximum incidence of $0.5 \%$. Recently, Al-Halal et al. reported that 3.3 cases of cervical cancer occurred per 100,000 births, and 130 cases of cervical intraepithelial neoplasia (CIN) were diagnosed per 100,000 births during pregnancy [1]. An increasing incidence of CIN among young Japanese women has been observed in recent years [2]. Fortunately, all pregnant women have multiple routine cytological examinations. Therefore, many asymptomatic women can be diagnosed at early stages of CIN.

A consensus exists on the diagnosis, evaluation and treatment of CIN in non-pregnant women [3], but such agreement does not exist for pregnant women. Indeed, the diagnosis of CIN during pregnancy raises questions regarding the management of this disease. Firstly, the interpretation of Pap smears obtained during pregnancy can be problematic as numerous physiological changes associated with the gravid state can lead to false positive results. Interestingly, it has been reported that CIN during pregnancy is likely to regress in the postpartum period [2].

In this study, we studied the progression of CIN in a cohort of pregnant women in line with the Guidelines for Obstetric Practice in Japan as a clinical practice. The aim of this study was to understand how CIN in pregnant women should be managed, and how the pathophysiological findings of CIN change in the postpartum period.

\section{SUBJECTS AND METHODS}

We conducted a retrospective analysis of the medical records of 24 patients who is all of the women diagnosed with CIN and managed in accordance with the Guidelines for Obstetric Practice in Japan at Ehime University Hospital between January 2008 and October 2012. Diagnoses and re-evaluations of disease status were made after cytological, colposcopic and histological examinations. Repeated cytological examinations were performed during pregnancy. All Pap smears were obtained with cotton swabs during pregnancy and with a cytobrush after delivery. Re-evaluations were performed 4 - 8 weeks after delivery. The mode of delivery was also recorded. 


\section{RESULTS}

The mean age of the patients was 29.5 years (range, 17 42 years). Four patients were diagnosed with CIN1 or CIN2 antenatally. Of these patients, all pathological lesions regressed or remained stable during the pregnancy, none of them progressed in the postpartum period. The cervical cytology and histology results during pregnancy and postpartum are shown in Table 1. Patient 4 had been referred with cytological results that were suspicious of microinvasion; she underwent conization at 15 weeks of gestation. The histological analysis of her cervical biopsy sample revealed CIN2.

Nine patients were diagnosed with severe dysplasia (CIN3) antenatally (Table 2). Of these patients, 5/9 (55\%) women had disease regression postnatally. Three patients underwent cesarean sections. Case 7 underwent cesarean section as the fetus was in the breech presentation. Case 10 underwent cervical conization at 16 weeks of gestation because of a suspected microinvasive cervical carcinoma. Histological examination of the conization confirmed carcinoma in situ (CIS) with a positive margin. She was followed-up using cervical cytology, which was suspicious of a microinvasive cervical carcinoma again at 36 weeks of gestation. At this point, she

Table 1. The status of 4 patients diagnosed with CIN1 or CIN2.

\begin{tabular}{ccccccc}
\hline Patient & Age & $\begin{array}{c}\text { Prenatal } \\
\text { cytology }\end{array}$ & $\begin{array}{c}\text { Prenatal } \\
\text { histology }\end{array}$ & Delivery Postpartum Postpartum \\
mode & cytology & histology \\
\hline 1 & 28 & I - IIIa & CIN1 & VD & I & \\
2 & 32 & IIIa & CIN1 & CS & IIIa & CIN1 \\
3 & 41 & III & CIN1 & CS & IIIa & CIN1 \\
4 & 17 & IIIa - V & CIN1-2 & VD & I - III & \\
\hline
\end{tabular}

VD: vaginal delivery; CS: caesarean section. underwent a cesarean section. The final histological diagnosis was CIS and a repeat conization was performed 2.5 months after delivery, which confirmed CIS with no positive margin.

In total, 11 patients were diagnosed with CIS (Table 3 ), of which four (36\%) women showed disease regression in the postpartum period. None of these patients required a cesarean section, and none of the lesions progressed to an invasive carcinoma during or after the pregnancy. All of the lesions that regressed did so within the first 3 months postnatally.

\section{DISCUSSION}

Over the past 20 years, the incidence of cervical cancer in young women aged 20 to 30 years has been increased, [4] largely as a result of an increase in the rate of human papillomavirus (HPV) infection in this generation. Also, the age of women at their first childbirth has been increased. Pregnancy offers young women the opportunity to have cervical cytological screening as part of the routine recommended examination, which may be the first time they have participated in cervical screening for cervical cancer. This could have led to the increase in the diagnostic rate of CIN during pregnancy.

Carcinoma of the cervix is currently the most common gynecological cancer found during pregnancy [5]. Recent reports have shown that the overall incidence of cervical cancer in pregnancy was $3.3-3.7$ per 100,000 pregnancies $[1,6]$, and pregnant women comprised $1 \%-3 \%$ in all patients with cervical cancer [7]. Cervical dysplasia during pregnancy has been reported in 0.7 per 100 pregnancies [8]. However, the incidence of cervical dysplasia is similar between pregnant and non-pregnant women [9]. In general, it is thought that pregnancy does not adversely influence the survival of patients with cervical cancer [10].

Table 2. The status of 9 patients diagnosed with CIN3.

\begin{tabular}{|c|c|c|c|c|c|c|c|c|}
\hline Patient & Age & $\begin{array}{l}\text { Prenatal } \\
\text { cytology }\end{array}$ & $\begin{array}{c}\text { Prenatal } \\
\text { histology }\end{array}$ & Delivery mode & $\begin{array}{l}\text { Postpartum } \\
\text { cytology }\end{array}$ & $\begin{array}{l}\text { Postpartum } \\
\text { histology }\end{array}$ & Operation & Last histology \\
\hline 5 & 32 & IIIa & CIN3 & VD & I - II & & & \\
\hline 6 & 22 & II - IIIb & CIN3 & VD & II & CIN1 & & \\
\hline 7 & 33 & IIIa - IIIb & CIN3 & $\mathrm{CS}$ & IIIa - IIIb & no disease & & \\
\hline 8 & 25 & IIIa & CIN3 & VD & IIIa & CIN2 & Ablation & CIN2 \\
\hline 9 & 29 & III & CIN3 & $\mathrm{CS}$ & II & CIN1 & & \\
\hline 10 & 34 & IIIb - IV & $\begin{array}{l}\text { Microinvasive } \\
\text { suspect }\end{array}$ & $\mathrm{CS}$ & IV & CIS & Conization & CIS \\
\hline 11 & 21 & IIIa - IIIb & CIN3 & VD & IIIb & CIN3 & Conization & CIS \\
\hline 12 & 26 & I - IIIb & $\mathrm{CN} 3$ & VD & IIIb & CIS & Conization & CIN3 \\
\hline 13 & 32 & IIIb - V & CIN3 & VD & IIIb & CIN3 & Conization & CIN3 \\
\hline
\end{tabular}

VD: vaginal delivery; CS: caesarean section. 
Table 3. The status of 11 patients diagnosed with CIS.

\begin{tabular}{|c|c|c|c|c|c|c|c|c|}
\hline Patient & Age & $\begin{array}{l}\text { Prenatal } \\
\text { cytology }\end{array}$ & $\begin{array}{c}\text { Prenatal } \\
\text { histology }\end{array}$ & Delivery mode & $\begin{array}{l}\text { Postpartum } \\
\text { cytology }\end{array}$ & $\begin{array}{l}\text { Postpartum } \\
\text { histology }\end{array}$ & Operation & Last histology \\
\hline 14 & 31 & I - IIIb & CIS & VD & $\mathrm{IV} \rightarrow \mathrm{II}$ & CIN1 & & \\
\hline 15 & 35 & IIIb & CIS & VD & IIIb & CIN3 & Conization & No lesion \\
\hline 16 & 35 & III - IV & CIS & VD & IV & CIN1 & Conization & CIN2 \\
\hline 17 & 35 & IIIb & CIS & VD & II & & & \\
\hline 18 & 34 & I - IIIb & CIS & VD & II & CIS & Conization & CIS \\
\hline 19 & 35 & IIIb & CIS & VD & IIIb & CIS & Conization & CIS \\
\hline 20 & 26 & IIIb & CIS & VD & III & CIN3 & Conization & CIS \\
\hline 21 & 28 & IV & CIS & VD & IIIb & CIN3 & Conization & CIN3 \\
\hline 22 & 33 & IIIb - IV & CIS & VD & $\mathrm{V}$ & CIS & Conization & CIN3 \\
\hline 23 & 21 & IIIb - IV & CIS & VD & $\mathrm{IIIb}$ & CIS & Conization & CIS \\
\hline 24 & 34 & IV & CIS & VD & IIIb & CIS & Conization & CIS \\
\hline
\end{tabular}

VD: vaginal delivery; CS: caesarean section.

As a result of the high estrogen state in pregnancy, the uterine cervix undergoes dramatic physiological changes. Therefore, the interpretation of Pap smears obtained during pregnancy can be problematic, leading to false positive results. Therefore, the sensitivity of this investigation in pregnancy ranges from only $70 \%$ to $84 \%$ [11]. Microscopic eversion of the transformation zone and exposure of columnar cells to the acidic vaginal $\mathrm{pH}$ can also causes squamous metaplasia, which may be falsely interpreted as dysplasia. The changes seen with AriasStella reaction may also resemble endocervical adenocarcinoma [12]. It is therefore essential to inform the cytopathologists who interpret Pap smears when the patient is pregnant.

The physiological changes of pregnancy also make the interpretation of colposcopy difficult if performed during pregnancy. The most important purpose of colposcopy is to exclude the presence of invasive cancer. However, pregnancy tends to exaggerate the colposcopic appearance of CIN, which might give rise to overdiagnosis. [13].

As a result of these reasons, the diagnosis of CIN during pregnancy poses several management problems, particularly in the decision of whether or not to treat the lesion during the pregnancy.

In this study, we managed the disease in accordance with the Guidelines for the Obstetric Practice in Japan. [14] As a result, no CIN lesion progressed to invasive carcinoma during pregnancy or after delivery. Additionally, there were no major complications in this cohort during pregnancy.

Some authors have reported high rates of regression of CIN postnatally, ranging from $47 \%$ to $70 \%$ [15-18]. Our data was in accordance with these previous findings, as the disease of 5/9 (55\%) patients diagnosed with severe dysplasia during pregnancy regressed postnatally. CIS is more likely to progress to invasive cancer. However, we observed that 4/11 (36\%) patients diagnosed with CIS had disease regression, and none developed invasive carcinoma either during pregnancy or postnatally. The epithelial and hormonal changes that occur during the postpartum phase, particularly in women who are breastfeeding, may mask lesions, which could account for the higher reported rates of regression reported $[15,19]$.

The route of delivery is also an important consideration. Some authors have reported a higher rate of regression of cervical dysplasia is associated with vaginal delivery compared to cesarean sections [20]. Coppola et al. refuted the hypothesis that the cervical trauma associated with delivery may induce regression in the CIN lesion, as they found no correlation between the route of delivery and the persistence of the disease [21]. We could not perform a statistical analysis regarding the type of delivery, as cesarean sections were only performed in three patients.

It is recommended that a re-evaluation using cytology and colposcopy should be performed $4-8$ weeks after delivery. Our data revealed that postpartum spontaneous regression observed within 3 months. On the other hand, Kaplan et al. reported that patients with antepartum lowgrade squamous intraepithelial lesion (LSILs) or highgrade squamous intraepithelial lesion (HSILs) have a high risk of disease recurrence 5 years later [22].

In conclusion, we demonstrated that the majority of CIN2 regressed or remained stable during pregnancy, On the other hand, CIN3 persisted during pregnancy and 
postnatally despite the route of delivery. As mentioned above, it is important that conservative antepartum management with postpartum re-evaluations, regardless of the route of the delivery, as we remain unable to predict which lesions are more likely to regress. Conization for persistent lesions is recommended after 3 months postpartum. However, the discussion is limited because of the small sample size. Further large-scale examination is needed.

\section{REFERENCES}

[1] Al-Halal, H., Kezouh, A. and Abenhaim, H.A. (2013) Incidence and obstetrical outcomes of cervical intraepithelial neoplasia and cervical cancer in pregnancy: A population-based study on 8.8 million births. Archives of Gynecology and Obstetrics, 287, 245-250. doi:10.1007/s00404-012-2475-3

[2] Ueda, Y., Enomoto, T., Miyatake, T., Yoshino, K., Fujita, M., Miyake, T., Fujiwara, K., Muraji, M., Kanagawa, T. and Kimura, T. (2009) Postpartum outcome of cervical intraepithelial neoplasia in pregnant women determined by route of delivery. Reproductive Sciences, 16, 10341039. doi: $10.1177 / 1933719109339349$

[3] Wright Jr., T.C., Massad, L.S., Dunton, C.J., Spitzer, M., Wilkinson, E.J. and Solomon, D. (2007) 2006 consensus guidelines for the management of women with cervical intraepithelial neoplasia or adenocarcinoma in situ. American Journal of Obstetrics and Gynecology, 197, 340345 .

[4] Ushijima, K.J. (2009) Current status of gynecologic cancer in Japan. Journal of Gynecologic Oncology, 20, 67-71.

[5] Method, M.W. and Brost, B.C. (1999) Management of cervical cancer in pregnancy. Seminars in Surgical Oncology, 16, 251-260. doi:10.1002/(SICI)1098-2388(199904/05)16:3<251::AID -SSU11>3.0.CO;2-4

[6] Smith, L.H., Danielsen, B., Allen, M.E. and Cress, R. (2003) Cancer associated with obstetric delivery: Results of linkage with the California cancer registry. American Journal of Obstetrics and Gynecology, 189, 1128-1135.

[7] Nguyen, C., Montz, F.J. and Bristow, R.E. (2000) Management of stage I cervical cancer in pregnancy. Obstetrical \& Gynecological Survey, 55, 633-643.

[8] Palle, C., Bangsbøll, S. and Andreasson, B. (2000) Cervical intraepithelial neoplasia in pregnancy. Acta Obstetricia et Gynecologica Scandinavica, 79, 306-310.

[9] Ueki, M., Ueda, M., Kumagai, K., Okamoto, Y., Noda, S. and Matsuoka, M. (1995) Cervical cytology and conservative management of cervical neoplasias during pregnancy. International Journal of Gynecological Pathology, 14, 63-69.

[10] Hopkins, M.P. and Morley, G.W. (1992) The prognosis and management of cervical cancer associated with pregnancy. Obstetrics \& Gynecology, 80, 9-13.

[11] Hunter, M.I., Monk, B.J. and Tewari, K.S. (2008) Cervi- cal neoplasia in pregnancy. Part 1: Screening and management of preinvasive disease. American Journal of $\mathrm{Ob}$ stetrics and Gynecology, 199, 3-9.

[12] Campion, M.J. and Sedlacek, T.V. (1993) Colposcopy in pregnancy. Obstetrics and Gynecology Clinics of North America, 20, 153-163.

[13] Ostergard, D.R. and Nieberg, R.K. (1979) Evaluation of abnormal cervical cytology during pregnancy with colposcopy. American Journal of Obstetrics and Gynecology, 134, 756-758.

[14] Minakami, H., Hiramatsu, Y., et al. (2011) Guidelines for obstetrical practice in Japan: Japan Society of Obstetrics and Gynecology (JSOG) and Japan Association of Obstetricians and Gynecologists (JAOG) 2011 edition. Journal of Obstetrics and Gynaecology Research, 37, 224-226.

[15] Vlahos, G., Rodolakis, A., Diakomanolis, E., Stefanidis, K., Haidopoulos, D., Abela, K., Georgountzos, V. and Michalas, S. (2002) Conservative management of cervical intraepithelial neoplasia $\left(\mathrm{CIN}_{2-3}\right)$ in pregnant women. Gynecologic and Obstetric Investigation, 54, 78-81. doi:10.1159/000067715

[16] Ahdoot, D., Van Nostrand, K.M., Nguyen, N.J., Tewari, D.S., Kurasaki, T., DiSaia, P.J. and Rose, G.S. (1998) The effect of route of delivery on regression of abnormal cervical cytologic findings in the postpartum period. American Journal of Obstetrics and Gynecology, 178, 1116-1120.

[17] Paraskevaidis, E., Koliopoulos, G., Kalantaridou, S., Pappa, L., Navrozoglou, I., Zikopoulos, K. and Lolis, D.E. (2002) Management and evolution of cervical intraepithelial neoplasia during pregnancy and postpartum. European Journal of Obstetrics, Gynecology, and Reproductive Biology, 104, 67-69.

[18] Serati, M., Uccella, S., Laterza, R.M., Salvatore, S., Beretta, P., Riva, C. and Bolis, P.F. (2008) Natural history of cervical intraepithelial neoplasia during pregnancy. Acta Obstetricia et Gynecologica Scandinavica, 87, 1296-1300. doi:10.1080/00016340802482986

[19] Yost, N.P., Santoso, J.T., McIntire, D.D. and Iliya, F.A. (1999) Postpartum regression rates of antepartum cervical intraepithelial neoplasia II and III lesions. Obstetrics and Gynecology, 93, 359-362.

[20] Ahdoot, D., Van Nostrand, K.M., Nguyen, N.J., Tewari, D.S., Kurasaki, T., DiSaia, P.J. and Rose, G.S. (1998) The effect of route of delivery on regression of abnormal cervical cytologic findings in the postpartum period. American Journal of Obstetrics and Gynecology, 178, 1116-1120.

[21] Coppola, A., Sorosky, J., Casper, R., Anderson, B. and Buller, R.E. (1997) The clinical course of cervical carcinoma in situ diagnosed during pregnancy. Gynecologic Oncology, 67, 162-165.

[22] Kaplan, K.J., Dainty, L.A., Dolinsky, B., Rose, G.S., Carlson, J., McHale, M. and Elkas, J.C. (2004) Prognosis and recurrence risk for patients with cervical squamous intraepithelial lesions diagnosed during pregnancy. Cancer, 102, 228-232. 